\title{
HTCSLQ : Hierarchical Tree Congestion Degree with Speed Sending and Sum Costs Link Quality Mechanism for Wireless Sensor Networks
}

\author{
Mbida Mohamed ${ }^{1}$ \\ Phd student \\ Department of Emerging Technologies Laboratory (LAVETE) \\ Faculty of Sciences and Technology Hassan 1st University \\ Settat, Morocco
}

\author{
Ezzati Abdellah ${ }^{2}$ \\ Professor \\ Department of Emerging Technologies Laboratory (LAVETE) \\ Faculty of Sciences and Technology Hassan 1st University \\ Settat, Morocco
}

\begin{abstract}
Wireless Sensor Network (wsn) performances have progressed over the last few years, aiming at expanding the lifetime nodes. Among the important studied parts on wsn is the congestion degree that can be identified by several algorithms such as HTAP (Hierarchical Tree Alternative Path), which is the target in the current study. It is important to mention that besides the variations observed between the four HTAP deployments of this classical algorithm ( detailed on the rest of paper ), there is a possibility that other factors, such as energy efficiency, network lifetime are affected by the node displacements in the HTAP phases ( in case of the route change caused by the isolated dead nodes to the sink ), which give the idea to reduce this undesirable problem by designing a new algorithm called Hierarchical Tree Congestion degree with Speed sending and Sum costs link Quality (HTCSLQ) by reducing the energy consumption, according to the choice of the lower Congestion degree, optimal Speed sending and Sum costs link Quality values from the source to destination.
\end{abstract}

Keywords-HTAP (Hierarchical Tree Alternative Path); Cd (congestion degree); Ss( speed of sending); SCVQL (Sum of costs variable Link quality); Wireless Sensor Network (wsn)

\section{INTRODUCTION}

Wsn nodes is an embedded system that links between sensors/actuators with different communications mechanisms, each node is characterized by autonomy and establish a collaboration with their neighbors in order to form the network and transmit data to the sink, for this reason it is important to use the management system in queues of wsn.

The queues management Causes problems in sequencing level of data, among those factors which affect negatively the performance of wsn is the congestion (the target of this study). There are many protocols in the literatures designed to control congestion wsn, as the HATP(Hierarchical tree Alternative Path ) protocol is most study and is designed to check and avoid the congestion under four differents layouts node placement (explained in the related work of this article ). In this article, a new congestion control protocol HTCSLQ are designed, which will corrects the overconsumption energy of problems displacements nodes compared to the execution of classic phases HTAP.This research requirement focuses on modeling a new Algorithm Congestion on WSN, with optimized energy consumption. The paper is organized as follows : Section II presentation of related work Section III description of HTCSLQ mechanisms to avoid the problems of displacements nodes. Section 4 demonstrates the proposed dynamic HTCSLQ algorithm. Section 5 provides simulation and comparative study with the classical HTAP using the Jprowler simulator, and Section 6 the conclusion.

\section{RELATED WORK}

In the literature HTAP is distributed and scalable framework with any type of network in order to reduce the level of congestion and make a safe transmission in each scenario, and designed to minimize packet Losses.This technique is based on the creation of alternative paths between the source and the sink, this establishment does not always make only the use of nodes in initial shorter path, its linear randomized choice of next hops (random selection of Congestion degree value in each elected path nodes ) ( Figure 1 ) involves and keep communication between nodes. 2) :

HTAP algorithm consists of four fundamental parts (Figure

> Flooding with level discovery functionality (FD): Through this step, each node locate its neighbors and renew its neighbor table, and every node is placed in levels between the source and the sink.

> Alternative Path Creation Algorithm (APC) (boolean congested or not ): In goal to avoid congestion, every congested node receiver is sending a notification packet congestion to inform the sender. Accordingly the sender node stops the transmission of packets to the congested node and finds another least congested node in its neighbor table in aim to maintain the transmission of data, and the creation of new roots is done to the sink.

The Hierarchical Tree Algorithm (HT): A hierarchical tree is created between the nodes, and became connected using the exchange of packets handshake,this packets contains also the state of congestion. This two algorithms combined build the Hierarchical Tree Alternative Path (HTAP) algorithm.

Handling of Powerless (Dead Nodes): 
The HTAP algorithm in case of the nodes going to lose its energy and directly isolated from the network and the tables of its neighbor nodes are updated.

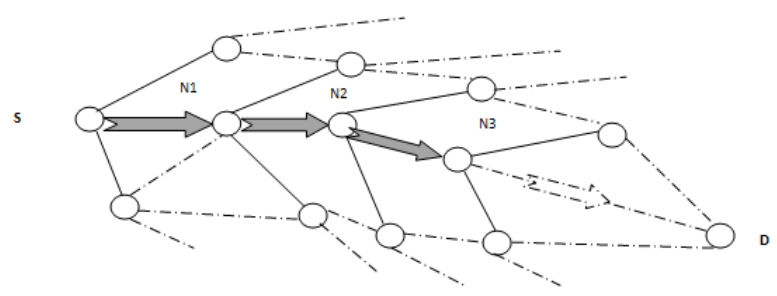

Fig. 1. Example of randomized and linear choice HTAP

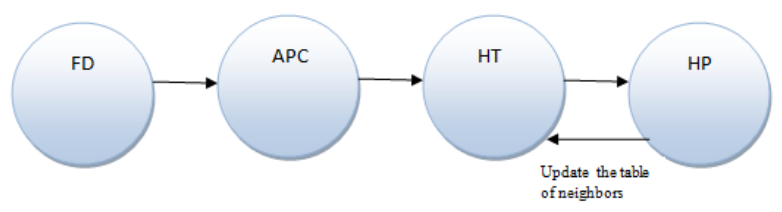

Fig. 2. HTAP algorithm functioning

\section{THE THEORICAL VIEW OF HTCSLQ}

HTCSLQ Algorithm are designed in order to be adaptable with any kind of network purpose is to widen the network life time and to increase its during the transmission data, The following phases describes its functioning (Figure 3).

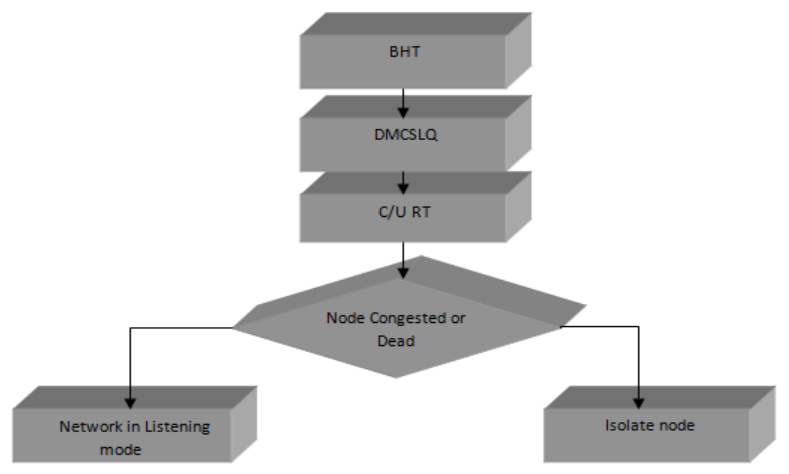

- BHT : Building of Hierarchical tree

- DMCSLQ : Detection and measurement of Cd, SS , SCVLQ of each node

- C/U RT : Construction or updating every root table with the three factors

Fig. 3. Architecture of HTCSLQ building Network

\section{A. Congestion deggree mechanism}

The calculation of Congestion Degree is the ratio between Local packet inter-arrival time and Local packet inter-service time. The measurement of the value of congestion Degree specify the rate of congestion of the network. the following formula presente the theorical calculation :

Congestion Degree $(\mathrm{Cd})=\mathrm{Ts} / \mathrm{Ta}$ Where $\mathrm{Ts}=$ local packet inter-service time, $\mathrm{Ta}=$ local packet inter-arrival time

\begin{tabular}{|l|}
\hline \multicolumn{1}{c|}{ Algorithm 1 : Cd calculation } \\
\hline Input : \\
Ts : local packet inter-service time \\
Ta : local packet inter-arrival time \\
Tree of nodes : TN \\
Node : $\mathrm{n}$ \\
Output: \\
Congestion degree value : CDV \\
for each ni $\varepsilon$ TN do \\
for each cdi of ni \\
Cdvi<==Tsi/Tai \\
End \\
End
\end{tabular}

\section{B. Speed of sending calculation}

When a node has a packet to be sent to the sink, it has to calculate the speed of the available nodes. Based on this estimation, the nodes which have the ability to send a packet according to the suitable speed. In order to do this, the sender is connected to sink node, for example the node $\mathrm{i}$ with coordinates (x i , y i) and the destination with $(\mathrm{x} \mathrm{d}, \mathrm{y} \mathrm{d})$, the progress of node $\mathrm{j}$ with location $(\mathrm{x} \mathrm{j}, \mathrm{y} j)$ is found by the projection of point $\mathrm{j}$ into line connecting $\mathrm{i}$ and $\mathrm{d}$ and is presented as $\mathrm{P}$ ij (Figure 4). The value of $\mathrm{P}$ ij is calculated with the formula : $\mathrm{P} \mathrm{ij}=\mathrm{D}$ ij* $\cos \alpha$

Where $\mathrm{D}$ ij is the distance between node $\mathrm{i}$ and $\mathrm{j}$ and $\alpha$ is the angle between nodes $\mathrm{ij}$ and id. These are computed as:

$D$ ij $=\sqrt{(x i-x j) 2+(y i-y j) 2}$ and $\alpha=$ $\arctan |\mathrm{m} 1-\mathrm{m} 2| /|1+\mathrm{m} 1 \mathrm{~m} 2|$

in which $\mathrm{m} 1$ and $\mathrm{m} 2$ are the sloop of line ij and line id respectively

$m 1=y j-y i / x j-x i, m 2=y d-y i / x d-x i$

The speed for node $\mathrm{j}$ is found as: Speed $\mathrm{ij}=\mathrm{P} \mathrm{ij} /$ delay $\mathrm{ij}$

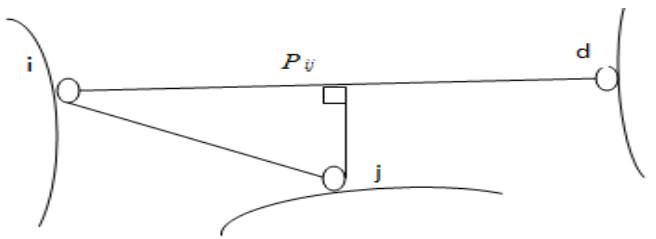

Fig. 4. Geometrical speed of sending calculation

The following algorithm describe the calculation :

\begin{tabular}{|l|}
\hline $\begin{array}{c}\text { Algorithm 2 : SS calculation of } \mathrm{jth} \\
\text { node }\end{array}$ \\
\hline Input : \\
Pnj : progress of node $\mathrm{j}$ \\
according to $\mathrm{n}$. \\
Delay nj : time to send \\
between node $\mathrm{n}$ and $\mathrm{j}$. \\
Tree of nodes : TN \\
Output: \\
Speed of Sending value : \\
SSV \\
\hline
\end{tabular}




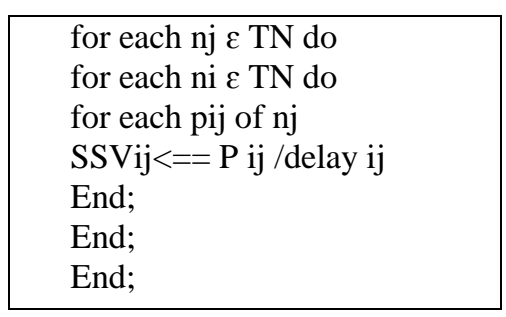

\section{Sum of Costs variable link quality calculation}

In this part the value of Link quality is calculated, in case : if there is three nodes connected ( $\mathrm{T}$ " source node" and $\mathrm{M}$ and $\mathrm{N}$ and $\mathrm{R}$ "final receiver" ), such as each node has its rooting table which contains the sum of costs variable link quality (SCVLQ) from the source to the sink, however this technique makes the Costs dynamic in the rooting table, as example if the node $\mathrm{N}$ receive the packet ( the simplified structure of packet is described in figure 6) from $M$, which contains the sum of costs part determined from the source to destination, its compared with the calculation of local sum costs to the same destination (at node $\mathrm{N}$ ), if it finds that the value of the SCVLQ of node N is smaller than the SCVLQ at node $M$, so it changes the value on the packet (figure5), else it changes the SCVLQ rooting table at node $\mathrm{N}$, however this mechanism provides a good transmission of packets with a best quality of links between nodes ( NB : This technique is used in sending packet phase, in this case all nodes will changes its SCVQL rooting table to the SCVQL packet after the choice of next hop explained in the rest of paper ).

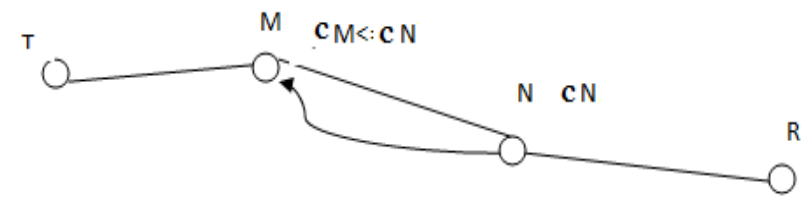

Fig. 5. Diagram of dynamic SCVLQ

The next algorithm illustrate the mechanism of SCVLQ:

\begin{tabular}{|l|}
\hline \multicolumn{1}{|c|}{ Algorithm 3 : SCVLQ calculation of jth node } \\
\hline Input : \\
Count Cost $\mathrm{X}$ : sumation of costs between source and sink in \\
routing table in Xth node . \\
Count Cost $\mathrm{Y}$ : sumation of costs between source and sink in \\
roting table of Yth node \\
Tree of nodes : TN \\
Minimal LQ cost : MLQC \\
Output: \\
Packet to next hop : PNH \\
for each ni $\varepsilon$ TN do \\
for each count cost $\mathrm{i}$ of ni \\
curent count costs $\mathrm{i}<==$ count cost $\mathrm{i}$ \\
End; \\
for each ni $\varepsilon$ TN do \\
if ( curent count costs $\mathrm{i}>$ curent count costs $\mathrm{i}+1)$ \\
curent count costs $\mathrm{i}<==$ curent count costs $\mathrm{i}+1$ \\
End; \\
Minimal LQ cost $<=$ curent count costs $\mathrm{i} ;$ \\
send PNH(Minimal LQ cost); \\
End;
\end{tabular}

\begin{tabular}{|c|c|c|c|c|c|c|c|}
\hline $\begin{array}{c}\text { Delimiter } \\
\text { Of onset } \\
\text { packet }\end{array}$ & ID & ASN & ADN & SCVLQ & DATA & $\ldots . . . . . . . . .$. & $\begin{array}{c}\text { Delimiter } \\
\text { Of the } \\
\text { end of } \\
\text { packet }\end{array}$ \\
\hline
\end{tabular}

ASN : Adress Source of Node

ADN : Adress Destination of Node

SCVLQ :Sum of costs variable Link Quality

Fig. 6. General structure of packet exchanged between nodes with ASVLQ

\section{D. $H T C S L Q$}

This algorithm are designed to correct the classical Algorithm of HTAP congestion, however it reduces the factors effects, such as energy efficiency, network lifetime which are affected by the node displacements, thus reduces more level of congestion better than the HTAP . The folowing algorithm present the functioning of HTCSLQ protocol using the 3 mechanisms :

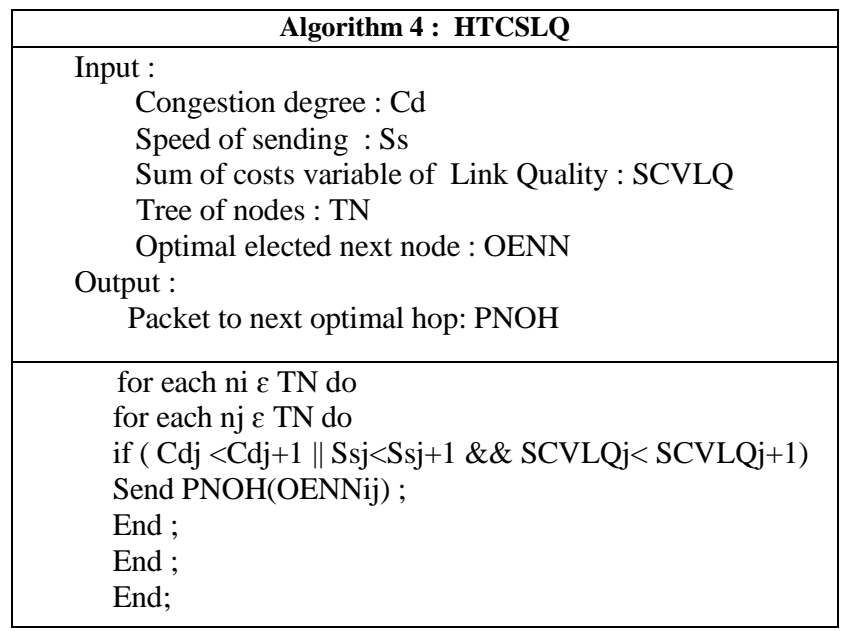

HTCSLQ also allows to quantify and measure the 3 variables ( Cd, Ss, SCVLQ) of each node ( Figure 7), after that it build the root from the source to the final destination, and also make choice of the elected next hops , the source send the data to this node and fill the SCVQL part of packet by the current SCVQL selected, however the HTAP allows just the detection of congestion, in order to isolate the nodes from the network.

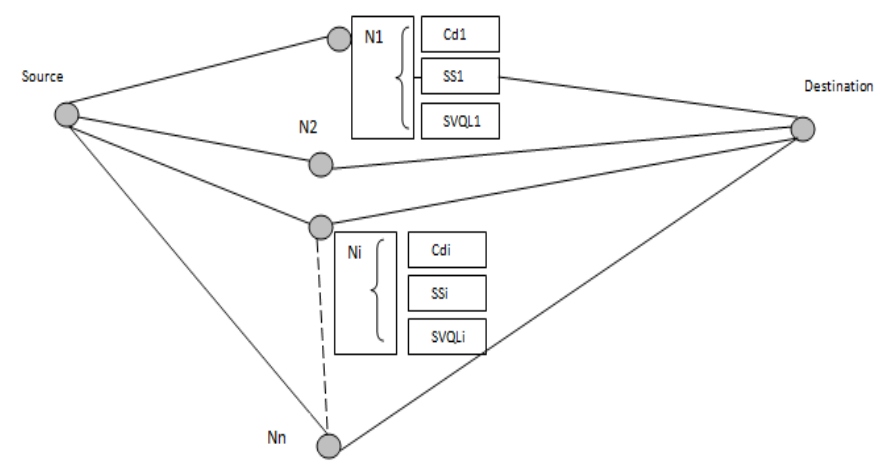

Fig. 7. Diagram HTCSLQ Measurements of the 3 mechanisms 


\section{SIMULATION AND RESULTS}

After a series of randomized simulations HTCSLQ and HTAP with the same model Tree ( from the root to the last leaf node of network ) and settings ( Table 1), on JProwler (Figure 8) the congestion control and characteristics of HTCSLQ algorithm is experimented with the simulation space which includes the deployment of the nodes in a $1000 \mathrm{~m} \mathrm{x}$ $1000 \mathrm{~m}$ grid.

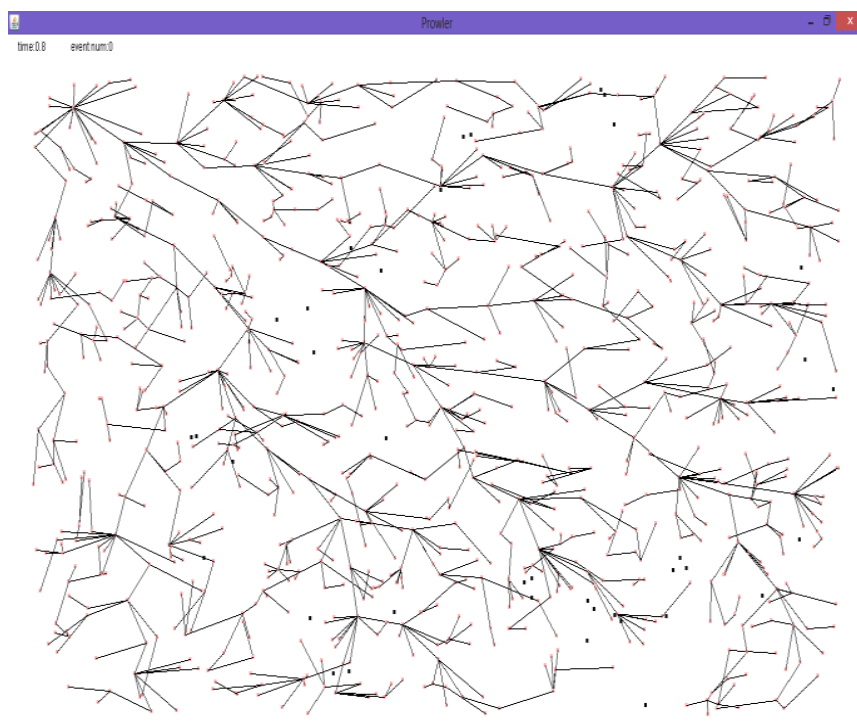

Fig. 8. Exemple of HTCSLQ Hirarchical tree in WSN

TABLE I. PARAMETRES OF SimUlations

\begin{tabular}{|l|l|}
\hline Simulation time & $20000 \mathrm{~ms}$ \\
\hline Max Radio Strength & 100 \\
\hline Type of Network & Grid \\
\hline Distibution node & uniform \\
\hline Radio strength cut off & $1 / 30$ \\
\hline Send transmission Time & $960 \mathrm{~ms}$ \\
\hline Noise variance & 0.025 \\
\hline $\begin{array}{l}\text { MaxAllowednoise on } \\
\text { sending }\end{array}$ & 5 \\
\hline Receiving Start SNR & 4.0 \\
\hline Corruption SNR & 2.0 \\
\hline Number of nodes & 1000 \\
\hline
\end{tabular}

\section{A. Experimental HTCSLQ mechanisms study}

In this part the Congestion degree, Speed of sending and sum of costs variable link quality of HTCSLQ is evaluated and compared with the HTAP algorithm, using by reference the encoding Java in JProwler Simulator:

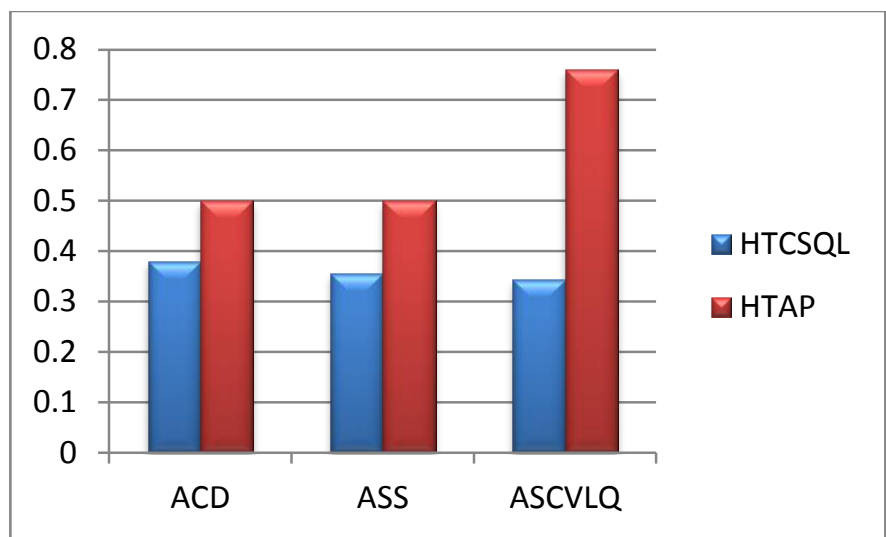

Indications:

\begin{tabular}{|ll|}
\hline ACD (coefficient) & : Average of congestion degree \\
\hline $\begin{array}{l}\text { ASVLQ real }=[\text { ASVQL } * 10] ~ \\
\text { variable Link quality }\end{array}$ & Average of Sum of costs \\
\hline ASS (kbits/s) & $:$ Average of Speed of Sending \\
\hline
\end{tabular}

Fig. 9. Comparative performance between HTCSLQ and HTAP

According to the comparative study ( Figure 9) the average congestion degree from the same source to the destination in the network of the algorithm HTCSLQ is optimal compared to the HTAP because the first detect the nodes which have a lower congestion then the average expected, however the HTAP detect only the nodes congested and isolate its . Also it is found that the ASS and ASCVLQ reached an average lower than has the HTAP, in order to find an optimal speed of data transmission and network stability with a minimum sum of costs. The life time of nodes becomes long because the HTCSLQ has the possibility to choose the nodes which are more efficient than other based on the 3 mechanisms ( $\mathrm{Cd}, \mathrm{SS}$ ,SCVLQ) in the case of rebuilding the network and isolation of congested or dead nodes .

\section{B. Most congested HTCSLQ and HTAP nodes}

Depending on the simulation under Jprowler, a function is encoded for showing the most congested nodes higher than the experimental average ( $\mathrm{cd}=0.6$ ) ( using the same configuration of Table 1), the following graphs represent the results for these two algorithms:

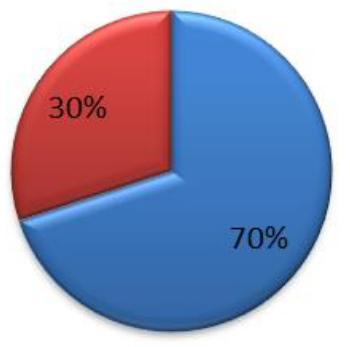

most congested nodes

no congested nodes

Fig. 10. Pourcentage of most HTAP congested nodes 


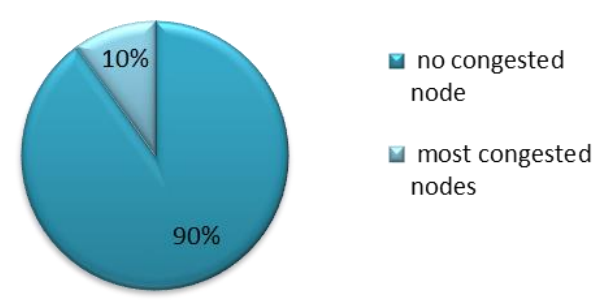

Fig. 11. Pourcentage of most HTCSLQ congested nodes

By Reference of successive simulations in the HTAP and HTCSLQ network on the same source to the destination, the linear and randomized choice of HTAP saturates the same nodes used during all simulations, however the HTCSLQ avoids pressure on the same node and uses the three mechanisms in goal to choose the best and optimal root in each simulation.

According to the two figures (Figure 10,11) the percentage of HTCSLQ most congested nodes which can be chosen as root from the same source to the destination ( from the root to the end of the tree structure in the current simulations ), reached a probability of $10 \%$, as long as the HTAP reached $70 \%$ ( Figure 10 and 11), and this led to deduce that the algorithm HTCSLQ during the reconstruction of the optimal root in case of every simulation from the same source to destination gives a longer life time to the network and energy efficiency of each node .

\section{CONCLUSION AND FUTURE WORK}

The HTCSLQ algorithm has succeeded in reducing the effects caused by the displacement of nodes ( phase of isolation dead nodes ) compared to the Classical HTAP , as said before the functioning of this Algorithm is resumed in two parts : measurement of the 3 variables $(\mathrm{Cd}, \mathrm{Ss}, \mathrm{SCVQL})$ in all networks nodes and choices of this optimal values in order to establish the less expensive root in terms of energy efficiency, network lifetime to the sink . The future work includes a study of fault tolerance integration and measurements, to design a new algorithm that can be compared with the performances of the current algorithm (HTCSLQ), and see if the integration and the optimal choice of this value in every nodes give more equilibrium energy and life time in the network.

\section{REFERENCES}

[1] C. Intanagonwiwat, R. Govindan, D. Estrin, J. Heidemann, and F. Silva, "Directed Diffusion for Wireless Sensor Networking," ACM/IEEE Transactions on Networking, vol. 11, no. 1, pp. 2-16, February 2002.

[2] M. Ishizuka and M. Aida, "Performance Study of Node Placement in Sensor Networks" In Proceedings of the 24th international Conference on Distributed Computing Systems

[3] C. Wang, K. Sohraby and B. Li, "SenTCP: A Hop-by-hop Congestion Control Protocol for Wireless Sensor Networks," In Proceedings of IEEE INFOCOM 2005 (Poster Paper), Miami, Florida, USA, Mar. 2005.

[4] S.-J. Park, R. Vedantham, R. Sivakumar, and I. F. Akyildiz, “A scalable approach for reliable downstream data delivery in wireless sensor networks," In Proceedings of ACM MobiHoc'04, May 24-26, 2004, Roppongi, Japan.
[5] J. Kang, Y. Zhang, and B. Nath, "End-to-End Channel Capacity Measurement for Congestion Control in Sensor Networks," In Proc. of the Second International Workshop on Sensor and Actor Network Protocols and Applications (SANPA 2004), August 2004

[6] C. Y. Wan, S. B. Eisenman, and A. T. Campbell, "CODA: Congestion Detection and Avoidance in Sensor Networks", In Proceedings of the ACM SenSys, November 2003.

[7] S. Tilaky, N. B. Abu-Ghazalehy and W. Heinzelmanz, "Infrastructure Tradeoffs for Sensor Networks," In Proc. of the 1st ACM international Workshop on Wireless Sensor Networks and Applications (WSNA '02), pp.49-58, Atlanta, GA, September 2002.

[8] C. Intanagonwiwat, R. Govindan, D. Estrin, J. Heidemann, and F. Silva, "Directed diffusion for wireless sensor networking," IEEE/ACM Transactions on Networking, vol. 11, no. 1, pp. 2-16, 2003.

[9] A. Cerpa, J. Elson, M. Hamilton, J. Zhao, D. Estrin, and L. Girod, "Habitat monitoring: Application driver for wireless communications technology," in SIGCOMM LA '01: Workshop on Data communication in Latin America and the Caribbean. New York, NY, USA: ACM, 2001, pp. $20-41$.

[10] C. Intanagonwiwat, R. Govindan, D. Estrin, J. Heidemann, and F. Silva, "Directed diffusion for wireless sensor networking," IEEE/ACM Transactions on Networking, vol. 11, no. 1, pp. 2-16, 2003.

[11] Cheng Wang, Changjun Jiang, Yunhao Liu, Senior Member, IEEE, Xiang-Yang Li, Senior Member, IEEE, and Shaojie Tang, IEEE TRANSACTIONS ON COMPUTERS, VOL. 63, NO. 6, JUNE 2014

[12] Ye, F.; Chen, A.; Lu, S.; Zhang, L. Scalable solution to minimum cost forwarding in large sensor networks. In Proceedings of 10th International Conference on Computer Communications andNetworks.

[13] Wang, X.; Bi, D.; Ding, L.; Wang, S. Agent collaborative target localization and classification in wireless sensor networks. Sensors 2007, 7, 1359-1386.

[14] Chen, J.; Li, S.; Sun, Y. Novel deployment schemes for mobile sensor networks. Sensors 2007,7, 2907-2919.

[15] Xu, Y.; Heidemann, J.; Estrin, D. Geography-informed energy conservation for ad hoc routing. In Proceedings of the 7th Annual ACM/IEEE International Conference on Mobile Computing and Networking.

[16] S. Yao, B. Mukherjee, S.J.B. Yoo, and S. Dixit, "All-Optical PacketSwitched Networks: A Study of Contention Resolution Schemes inan Irregular Mesh Network with Variable-Sized Packets," Proceedings, SPIE OptiComm 2000, Dallas, TX, pp.235-246, Oct. 2000.

[17] K.K. Ramakrishnan and Raj Jain, "A Binary Feedback Scheme for Congestion Avoidance in Computer Networks," ACM Transactions on Computer Systems, vol.8, no.2, pp.158-181, May 1990.

[18] Guru P.V. Thodime, Vinod M. Vokkarane, and Jason P. Jue The University of Texas at Dallas, Richardson," Dynamic Congestion-Based Load Balanced Routing in Optical Burst-Switched Networks ",USA 2013

[19] Kamal Kumar Sharma, Dr. Harbhajan Singh and Dr. R.B Patel "A Hop by Hop Congestion Control Protocol to Mitigate Traffic Contention in Wireless Sensor Networks" in Proceedings of International Journal of Computer Theory and Engineering, Vol.2, No.6, pp 1793-8201, December, 2010.

[20] Basaran, kyoung-Don Kang, Mehmet H. Suzer "Hop-by-Hop Congestio Control and Load Balancing in Wireless Sensor Networks", in proceedings of 2010 IEEE 35th conference on Local Computer Networks, pp. 448-455, 2010.

[21] Chieh-Yih Wan, Shane B. Eisenman,Andrew T. Campbell,” EnergyEfficient Congestion Detection and Avoidance in Sensor Networks ",in proceedings of ACM Transactions on Sensor Networks, Vol. 7, No. 4, Article 32,pp.32.1-32.31 2011.

[22] Babak Namazi*, Karim Faez** Department of Electrical Engineering, Amirkabir University of Technology," Energy-Efficient MultiSPEED Routing Protocol for Wireless Sensor Networks", International Journal of Electrical and Computer Engineering (IJECE)Vol.3,No.2,April2013 